\title{
Ultrastructural Study of Interactions between Phytophthora fragariae and a Biological Control Agent, Streptomyces hygroscopicus var. geldanus
}

\author{
M. Paquet*, S. Agbessi**, C. Beaulieu** and P. M. Charest* \\ *Université Laval, Dept. de Phytologie, Québec, QC, Canada G1K 7P4 \\ **Université de Sherbrooke, Dept. de Biologie, Sherbrooke, QC, Canada J1K 2R1
}

Raspberry root rot caused by Phytophthora fragariae var. rubi (PFR) is a worldwide disease that causes important economic losses. Once the pathogen is introduced in a field, yield loss and damages are severe and the entire crop can be totally destroyed in less than two years. At the present time, there are no chemical treatments that are recognized effective in the control of the disease as well as safe for the environment. The use of biological control agents proved to be an important alternative approach for the control of several plant pathogens. Among those agents, Actinomycetes are a group of microorganisms that is ubiquitous in the soil. Among 200 isolates of Streptomycetes screened for their ability to control PF, thirteen were selected as potential biocontrol candidates. One specific candidate, Streptomyces hygroscopicus sbsp. geldanus, (SHG), has been fully characterized (strain EF-76) and demonstrated strong abilities to control PFR in vitro or on raspberry plants in growth chambers and greenhouse experiments [1,2]. EF-76 was shown to secrete both glucanases and geldanamycin. These compounds might be responsible for PF growth inhibition. Antagonistic effects caused by SHG on hyphae of PFR were periodically observed during an in vitro experiment where PF was incubated in confrontation with EF-76.

Transmission electron microscopy studies revealed that the plasma membrane of PFR appeared strongly undulated and retracted only $2 \mathrm{~h}$ after the confrontation with SHG (Fig. 2). Four hours after treatment, cytoplasmic and organelle disorganization was observed (Fig. 3); then hyphae of PFR appeared moribund $8 \mathrm{~h}$ after co-cultivation. Control experiments were carried out on PFR cultures treated with $2 \mathrm{ml}$ of sterile culture liquid medium (Fig. 1). To verify that the main antagonistic mechanism was antibiosis, a second experiment was carried out where $15 \mathrm{mg}$ of the extracted geldanamycin dissolved in $2 \mathrm{ml}$ of sterile YME liquid medium was added to a 5-days old liquid culture of PFR. In the latter case, the same ultratructural and cellular disorganization features were observed in cells of PFR. We propose that the secretion of the antibiotic geldanamycin could be one of the factors that contribute to the control of this pathogen.

References

[1] Valois, D. et al. 1996. Glucanolytic actinomycetes antagonistic to Phytophthora fragariae var. rubi, the causal agent of raspberry root rot. Applied and Environmental Microbiology 62:16301635

[2] Toussaint, V. et al. 1997. Characterization of actinomycetes antagonistic to Phytophthora fragariae var. rubi, the causal agent of raspberry root rot. Phytoprotection 78:43-51 


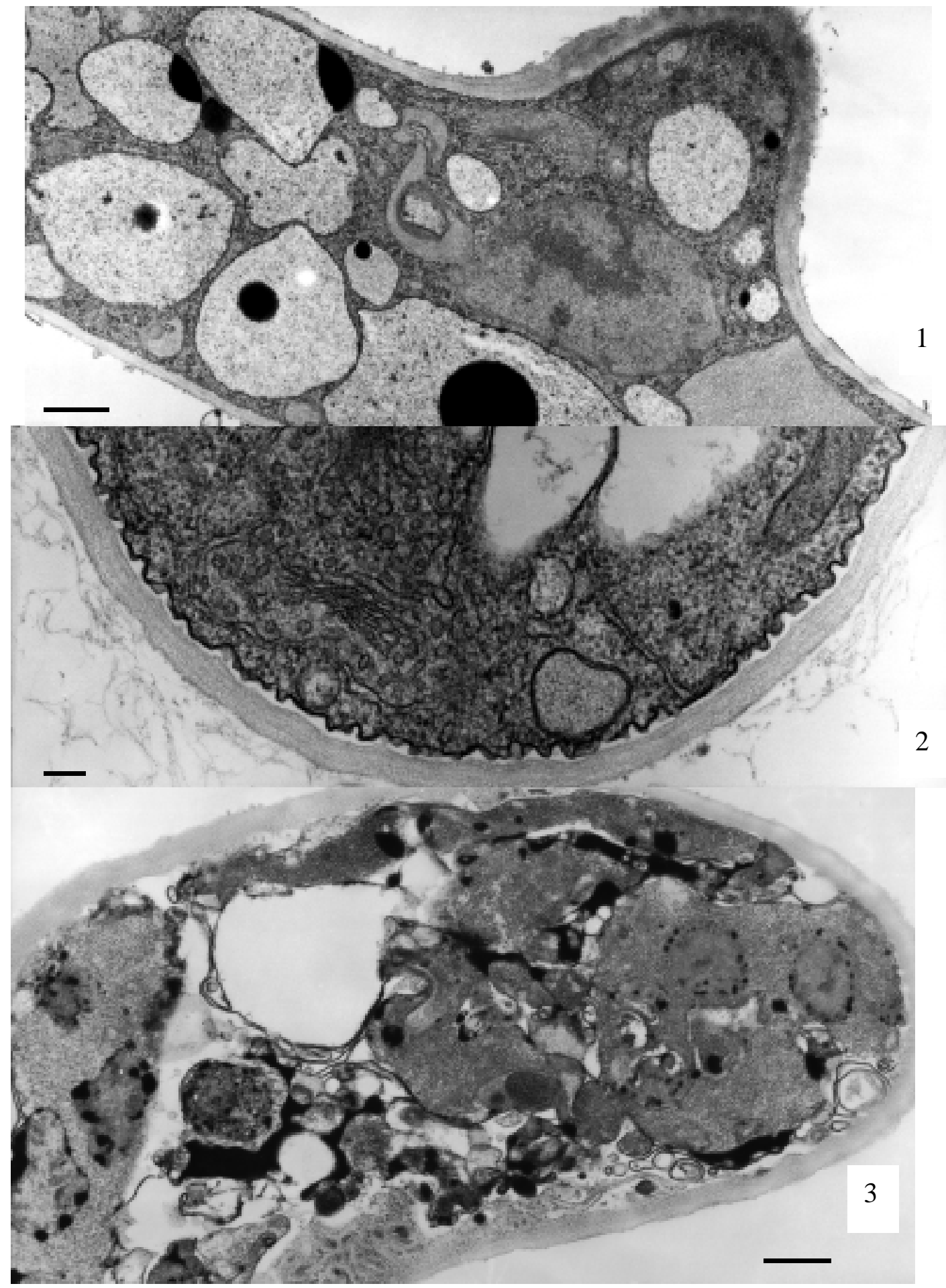

Figure 1: Control hyphae of Phytophthora fragariae (PFR) showing a dense granulated cytoplasm and typical ultrastructure. Scale bar $=100 \mathrm{~nm}$. Figure 2. Hyphae of PFR after a $2 \mathrm{~h}$ incubation with SHG. The plasmalemma is undulated and retracted from the cell wall. Scale bar $=200 \mathrm{~nm}$. Figure 3: Hyphae of PFR after a $8 \mathrm{~h}$ incubation with SHG. The plasmalemma is disrupted and the cytoplasm is strongly disorganized. Scale Bar $=250 \mathrm{~nm}$. 